\title{
Nonlocal Impulsive Cauchy Problems for Evolution Equations
}

\author{
Jin Liang' ${ }^{1}$ and Zhenbin Fan ${ }^{1,2}$ \\ ${ }^{1}$ Department of Mathematics, Shanghai Jiao Tong University, Shanghai 200240, China \\ ${ }^{2}$ Department of Mathematics, Changshu Institute of Technology, Suzhou, Jiangsu 215500, China
}

Correspondence should be addressed to Jin Liang, jinliang@sjtu.edu.cn

Received 17 October 2010; Accepted 19 November 2010

Academic Editor: Toka Diagana

Copyright (c) 2011 J. Liang and Z. Fan. This is an open access article distributed under the Creative Commons Attribution License, which permits unrestricted use, distribution, and reproduction in any medium, provided the original work is properly cited.

Of concern is the existence of solutions to nonlocal impulsive Cauchy problems for evolution equations. Combining the techniques of operator semigroups, approximate solutions, noncompact measures and the fixed point theory, new existence theorems are obtained, which generalize and improve some previous results since neither the Lipschitz continuity nor compactness assumption on the impulsive functions is required. An application to partial differential equations is also presented.

\section{Introduction}

Impulsive equations arise from many different real processes and phenomena which appeared in physics, chemical technology, population dynamics, biotechnology, medicine, and economics. They have in recent years been an object of investigations with increasing interest. For more information on this subject, see for instance, the papers (cf., e.g., [1-6]) and references therein.

On the other hand, Cauchy problems with nonlocal conditions are appropriate models for describing a lot of natural phenomena, which cannot be described using classical Cauchy problems. That is why in recent years they have been studied by many researchers (cf., e.g., [4, 7-12] and references therein).

In [4], the authors combined the two directions and studied firstly a class of nonlocal impulsive Cauchy problems for evolution equations by investigating the existence for mild (in generalized sense) solutions to the problems. In this paper, we study further the existence of solutions to the following nonlocal impulsive Cauchy problem for evolution equations: 


$$
\begin{gathered}
\frac{\mathrm{d}}{\mathrm{d} t}[u(t)+F(t, u(t))]+A u(t)=f(t, u(t)), \quad 0 \leq t \leq K, t \neq t_{i}, \\
u(0)+g(u)=u_{0}, \\
\Delta u\left(t_{i}\right)=I_{i}\left(u\left(t_{i}\right)\right), \quad i=1,2, \ldots, p, 0<t_{1}<t_{2}<\cdots<t_{p}<K,
\end{gathered}
$$

where $-A: D(A) \subseteq X \rightarrow X$ is the infinitesimal generator of an analytic semigroup $\{T(t) ; t \geq$ $0\}$ and $X$ is a real Banach space endowed with the norm $\|\cdot\|$,

$$
\begin{gathered}
\Delta u\left(t_{i}\right)=u\left(t_{i}^{+}\right)-u\left(t_{i}^{-}\right), \\
\left(u\left(t_{i}^{+}\right)=\lim _{t \rightarrow t_{i}^{+}} u(t), u\left(t_{i}^{-}\right)=\lim _{t \rightarrow t_{i}^{-}} u(t)\right),
\end{gathered}
$$

$F, f, g, I_{i}$ are given continuous functions to be specified later.

By going a new way, that is, by combining operator semigroups, the techniques of approximate solutions, noncompact measures, and the fixed point theory, we obtain new existence results for problem (1.1), which generalize and improve some previous theorems since neither the Lipschitz continuity nor compactness assumption on the impulsive functions is required in the present paper.

The organization of this work is as follows. In Section 2, we recall some definitions, and facts about fractional powers of operators, mild solutions and Hausdorff measure of noncompactness. In Section 3, we give the existence results for problem (1.1) when the nonlocal item and impulsive functions are only assumed to be continuous. In Section 4, we give an example to illustrate our abstract results.

\section{Preliminaries}

Let $(X,\|\cdot\|)$ be a real Banach space. We denote by $C([0, K], X)$ the space of $X$-valued continuous functions on $[0, K]$ with the norm

$$
\|u\|=\max \{\|u(t)\| ; t \in[0, K]\},
$$

and by $L^{1}([0, K], X)$ the space of $X$-valued Bochner integrable functions on $[0, K]$ with the norm $\|f\|_{L^{1}}=\int_{0}^{K}\|f(t)\| \mathrm{d} t$. Let

$$
\begin{gathered}
\operatorname{PC}([0, K], X):=\left\{u:[0, K] \rightarrow X ; u(t) \text { is continuous at } t \neq t_{i}, \text { left continuous at } t=t_{i},\right. \\
\text { and the right limit } \left.u\left(t_{i}^{+}\right) \text {exists for } i=1,2, \ldots, p\right\} .
\end{gathered}
$$

It is easy to check that $\mathrm{PC}([0, K], X)$ is a Banach space with the norm

$$
\|u\|_{\mathrm{PC}}=\sup _{t \in[0, K]}\|u(t)\|
$$


In this paper, for $r>0$, let $B_{r}:=\{x \in X ;\|x\| \leq r\}$ and

$$
W_{r}:=\left\{u \in \mathrm{PC}([0, K], X) ; u(t) \in B_{r}, \forall t \in[0, K]\right\} .
$$

Throughout this paper, we assume the following.

(H1) The operator $-A: D(A) \subseteq X \rightarrow X$ is the infinitesimal generator of a compact analytic semigroup $\{T(t): t \geq 0\}$ on Banach space $X$ and $0 \in \rho(A)$ (the resolvent set of $A$ ).

In the remainder of this work, $M:=\sup _{0 \leq t \leq K}\|T(t)\|<\infty$.

Under the above conditions, it is possible to define the fractional power $A^{\alpha}: D\left(A^{\alpha}\right) \subset$ $X \rightarrow X, 0<\alpha<1$, of $A$ as closed linear operators. And it is known that the following properties hold.

Theorem 2.1 (see [13, Pages 69-75]). Let $0<\alpha<1$ and assume that (H1) holds. Then,

(1) $D\left(A^{\alpha}\right)$ is a Banach space with the norm $\|x\|_{\alpha}=\left\|A^{\alpha} x\right\|$ for $x \in D\left(A^{\alpha}\right)$,

(2) $T(t): X \rightarrow D\left(A^{\alpha}\right)$ for $t>0$,

(3) $A^{\alpha} T(t) x=T(t) A^{\alpha} x$ for $x \in D\left(A^{\alpha}\right)$ and $t \geq 0$,

(4) for every $t>0, A^{\alpha} T(t)$ is bounded on $X$ and there exists $C_{\alpha}>0$ such that

$$
\left\|A^{\alpha} T(t)\right\| \leq \frac{C_{\alpha}}{t^{\alpha}}, \quad 0<t \leq K,
$$

(5) $A^{-\alpha}$ is a bounded linear operator in $X$ with $D\left(A^{\alpha}\right)=\operatorname{Im}\left(A^{-\alpha}\right)$,

(6) if $0<\alpha<\beta \leq 1$, then $D\left(A^{\beta}\right) \hookrightarrow D\left(A^{\alpha}\right)$.

We denote by $X_{\alpha}$ that the Banach space $D\left(A^{\alpha}\right)$ endowed the graph norm from now on.

Definition 2.2. A function $u \in \mathrm{PC}([0, K], X)$ is said to be a mild solution of $(1.1)$ on $[0, K]$ if the function $s \rightarrow A T(t-s) F(s, u(s))$ is integrable on $[0, t)$ for all $t \in[0, K)$ and the following integral equation is satisfied:

$$
\begin{aligned}
u(t)= & T(t)\left[u_{0}+F(0, u(0))-g(u)\right]-F(t, u(t))+\int_{0}^{t} A T(t-s) F(s, u(s)) \mathrm{d} s \\
& +\int_{0}^{t} T(t-s) f(s, u(s)) \mathrm{d} s+\sum_{0<t_{i}<t} T\left(t-t_{i}\right) I_{i}\left(u\left(t_{i}\right)\right), \quad 0 \leq t \leq K .
\end{aligned}
$$

To discuss the compactness of subsets of $\mathrm{PC}([0, K], X)$, we let $t_{0}=0, t_{p+1}=K$,

$$
J_{0}=\left(t_{0}, t_{1}\right], J_{1}=\left(t_{1}, t_{2}\right], \ldots, J_{p}=\left(t_{p}, t_{p+1}\right] .
$$

For $D \subseteq P C([0, K], X)$, we denote by $\left.D\right|_{\bar{J}_{i}}$ the set

$$
\left.D\right|_{\bar{J}_{i}}=\left\{u \in C\left(\left[t_{i}, t_{i+1}\right], X\right) ; u\left(t_{i}\right)=v\left(t_{i}^{+}\right), u(t)=v(t), t \in J_{i}, v \in D\right\},
$$

$i=0,1,2, \ldots, p$. Then it is easy to see that the following result holds. 
Lemma 2.3. A set $D \subseteq P C([0, K], X)$ is precompact in $P C([0, K], X)$ if and only if the set $\left.D\right|_{\bar{J}_{i}}$ is precompact in $C\left(\left[t_{i}, t_{i+1}\right], X\right)$ for every $i=0,1,2, \ldots, p$.

Next, we recall that the Hausdorff measure of noncompactness $\alpha(\cdot)$ on each bounded subset $\Omega$ of Banach space $Y$ is defined by

$$
\alpha(\Omega)=\inf \{\varepsilon>0 ; \Omega \text { has a finite } \varepsilon \text {-net in } Y\} .
$$

Some basic properties of $\alpha(\cdot)$ are given in the following Lemma.

Lemma 2.4 (see [14]). Let $Y$ be a real Banach space and let $B, C \subseteq Y$ be bounded. Then,

(1) $B$ is precompact if and only if $\alpha(B)=0$;

(2) $\alpha(B)=\alpha(\bar{B})=\alpha(\operatorname{conv} B)$, where $\bar{B}$ and $\operatorname{conv} B$ mean the closure and convex hull of $B$, respectively;

(3) $\alpha(B) \leq \alpha(C)$ when $B \subseteq C$;

(4) $\alpha(B+C) \leq \alpha(B)+\alpha(C)$, where $B+C=\{x+y ; x \in B, y \in C\}$;

(5) $\alpha(B \cup C) \leq \max \{\alpha(B), \alpha(C)\}$;

(6) $\alpha(\lambda B)=|\lambda| \alpha(B)$ for any $\lambda \in R$;

(7) let $Z$ be a Banach space and $Q: D(Q) \subseteq Y \rightarrow Z$ Lipschitz continuous with constant $k$. Then $\alpha(Q B) \leq k \alpha(B)$ for all $B \subseteq D(Q)$ being bounded.

We note that a continuous map $Q: W \subseteq Y \rightarrow Y$ is an $\alpha$-contraction if there exists a positive constant $k<1$ such that $\alpha(Q C) \leq k \alpha(C)$ for all bounded closed $C \subseteq W$.

Lemma 2.5 (see Darbo-Sadovskii's fixed point theorem in [14]). If $W \subseteq Y$ is bounded closed and convex, and $Q: W \rightarrow W$ is an $\alpha$-contraction, then the map $Q$ has at least one fixed point in $W$.

\section{Main Results}

In this section, by using the techniques of approximate solutions and fixed points, we establish a result on the existence of mild solutions for the nonlocal impulsive problem (1.1) when the nonlocal item $g$ and the impulsive functions $I_{i}$ are only assumed to be continuous in $\mathrm{PC}([0, K], X)$ and $X$, respectively.

In practical applications, the values of $u(t)$ for $t$ near zero often do not affect $g(u)$. For example, it is the case when

$$
g(u)=\sum_{j=1}^{q} c_{j} u\left(s_{j}\right), \quad 0<s_{1}<s_{2}<\cdots<s_{q}<K .
$$

So, to prove our main results, we introduce the following assumptions.

(H2) $g: \mathrm{PC}([0, K], X) \rightarrow X$ is a continuous function, and there is a $\delta \in\left(0, t_{1}\right)$ such that $g(u)=g(v)$ for any $u, v \in \operatorname{PC}([0, K], X)$ with $u(s)=v(s), s \in[\delta, K]$. Moreover, there exist $L_{1}, L_{1}^{\prime}>0$ such that $\|g(u)\| \leq L_{1}\|u\|_{\mathrm{PC}}+L_{1}^{\prime}$ for any $u \in \mathrm{PC}([0, K], X)$. 
(H3) There exists a $\beta \in(0,1)$ such that $F:[0, K] \times X \rightarrow X_{\beta}$ is a continuous function, and $F(\cdot, u(\cdot))=F(\cdot, v(\cdot))$ for any $u, v \in \operatorname{PC}([0, K], X)$ with $u(s)=v(s), s \in[\delta, K]$. Moreover, there exist $L_{2}, L_{3}>0$ such that

$$
\left\|A^{\beta} F\left(t, x_{1}\right)-A^{\beta} F\left(t, x_{2}\right)\right\| \leq L_{2}\left\|x_{1}-x_{2}\right\|
$$

for any $0 \leq t \leq K, x_{1}, x_{2} \in X$, and

$$
\left\|A^{\beta} F(t, x)\right\| \leq L_{3}(\|x\|+1)
$$

for any $0 \leq t \leq K, x \in X$.

(H4) The function $f(t, \cdot): X \rightarrow X$ is continuous a.e. $t \in[0, K]$; the function $f(\cdot, x)$ : $[0, K] \rightarrow X$ is strongly measurable for all $x \in X$. Moreover, for each $l \in \mathbb{N}$, there exists a function $\rho_{l} \in L^{1}([0, K], \mathbb{R})$ such that $\|f(t, x)\| \leq \rho_{l}(t)$ for a.e. $t \in[0, K]$ and all $x \in B_{l}$, and

$$
\gamma:=\liminf _{l \rightarrow \infty} \frac{1}{l} \int_{0}^{K} \rho_{l}(s) \mathrm{d} s<\infty
$$

(H5) $I_{i}: X \rightarrow X$ is continuous for every $i=1,2, \ldots, p$, and there exist positive numbers $L_{4}, L_{4}^{\prime}$ such that $\left\|I_{i}(x)\right\| \leq L_{4}\|x\|+L_{4}^{\prime}$ for any $x \in X$ and $i=1,2, \ldots, p$.

We note that, by Theorem 2.1, there exist $M_{0}>0$ and $C_{1-\beta}>0$ such that $M_{0}=\left\|A^{-\beta}\right\|$ and

$$
\left\|A^{1-\beta} T(t)\right\| \leq \frac{C_{1-\beta}}{t^{1-\beta}}, \quad 0<t \leq K .
$$

For simplicity, in the following we set $L=\max \left\{L_{1}, L_{2}, L_{3}, L_{4}\right\}$ and will substitute $L_{1}, L_{2}, L_{3}, L_{4}$ by $L$ below.

Theorem 3.1. Let (H1)-(H5) hold. Then the nonlocal impulsive Cauchy problem (1.1) has at least one mild solution on $[0, K]$, provided

$$
L_{0}=M\left(L+M_{0} L+\gamma+p L\right)+M_{0} L+\frac{L C_{1-\beta} K^{\beta}}{\beta}<1 .
$$

To prove the theorem, we need some lemmas. Next, for $n \in \mathbb{N}$, we denote by $Q_{n}$ the maps $Q_{n}: \mathrm{PC}([0, K], X) \rightarrow \mathrm{PC}([0, K], X)$ defined by

$$
\begin{aligned}
\left(Q_{n} u\right)(t)= & T(t)\left[u_{0}+F(0, u(0))-T\left(\frac{1}{n}\right) g(u)\right]-F(t, u(t))+\int_{0}^{t} A T(t-s) F(s, u(s)) \mathrm{d} s \\
& +\int_{0}^{t} T(t-s) f(s, u(s)) \mathrm{d} s+\sum_{0<t_{i}<t} T\left(t-t_{i}\right) T\left(\frac{1}{n}\right) I_{i}\left(u\left(t_{i}\right)\right), \quad 0 \leq t \leq K .
\end{aligned}
$$


In addition, we introduce the decomposition $Q_{n}=Q_{n 1}+Q_{n 2}+Q_{n 3}+Q_{n 4}$, where

$$
\begin{aligned}
& \left(Q_{n 1} u\right)(t)=T(t)\left[u_{0}-T\left(\frac{1}{n}\right) g(u)\right] \\
& \left(Q_{n 2} u\right)(t)=\sum_{0<t_{i}<t} T\left(t-t_{i}\right) T\left(\frac{1}{n}\right) I_{i}\left(u\left(t_{i}\right)\right) \\
& \left(Q_{n 3} u\right)(t)=T(t) F(0, u(0))-F(t, u(t))+\int_{0}^{t} A T(t-s) F(s, u(s)) \mathrm{d} s \\
& \left(Q_{n 4} u\right)(t)=\int_{0}^{t} T(t-s) f(s, u(s)) \mathrm{d} s
\end{aligned}
$$

for $u \in \mathrm{PC}([0, K], X)$ and $t \in[0, K]$.

Lemma 3.2. Assume that all the conditions in Theorem 3.1 are satisfied. Then for any $n \geq 1$, the map $Q_{n}$ defined by (3.7) has at least one fixed point $u_{n} \in P C([0, K], X)$.

Proof. To prove the existence of a fixed point for $Q_{n}$, we will use Darbu-Sadovskii's fixed point theorem.

Firstly, we prove that the map $Q_{n 3}$ is a contraction on $\mathrm{PC}([0, K], X)$. For this purpose, let $u_{1}, u_{2} \in \mathrm{PC}([0, K], X)$. Then for each $t \in[0, K]$ and by condition (H3), we have

$$
\begin{aligned}
&\left\|\left(Q_{n 3} u_{1}\right)(t)-\left(Q_{n 3} u_{2}\right)(t)\right\| \\
& \leq M\left\|F\left(0, u_{1}(0)\right)-F\left(0, u_{2}(0)\right)\right\|+\left\|F\left(t, u_{1}(t)\right)-F\left(t, u_{2}(t)\right)\right\| \\
& \quad+\int_{0}^{t}\left\|A T(t-s)\left[F\left(s, u_{1}(s)\right)-F\left(s, u_{2}(s)\right)\right]\right\| \mathrm{d} s \\
& \leq M\left\|A^{-\beta} A^{\beta} F\left(0, u_{1}(0)\right)-A^{-\beta} A^{\beta} F\left(0, u_{2}(0)\right)\right\|+\left\|A^{-\beta} A^{\beta} F\left(t, u_{1}(t)\right)-A^{-\beta} A^{\beta} F\left(t, u_{2}(t)\right)\right\| \\
& \quad+\int_{0}^{t}\left\|A^{1-\beta} T(t-s)\left[A^{\beta} F\left(s, u_{1}(s)\right)-A^{\beta} F\left(s, u_{2}(s)\right)\right]\right\| \mathrm{d} s \\
& \leq M M_{0} L\left\|u_{1}-u_{2}\right\|+M_{0} L\left\|u_{1}(t)-u_{2}(t)\right\|+\int_{0}^{t} \frac{C_{1-\beta}}{(t-s)^{1-\beta}} L\left\|u_{1}(s)-u_{2}(s)\right\| \mathrm{d} s .
\end{aligned}
$$

Thus,

$$
\left\|Q_{n 3} u_{1}-Q_{n 3} u_{2}\right\|_{\mathrm{PC}} \leq\left[(M+1) M_{0} L+\frac{L C_{1-\beta} K^{\beta}}{\beta}\right]\left\|u_{1}-u_{2}\right\|,
$$

which implies that $Q_{n 3}$ is a contraction by condition (3.6). 
Secondly, we prove that $Q_{n 4}, Q_{n 1}, Q_{n 2}$ are completely continuous operators. Let $\left\{u_{m}\right\}_{m=1}^{+\infty}$ be a sequence in $\mathrm{PC}([0, K], X)$ with

$$
\lim _{m \rightarrow \infty} u_{m}=u
$$

in $\mathrm{PC}([0, K], X)$. By the continuity of $f$ with respect to the second argument, we deduce that for each $s \in[0, K], f\left(s, u_{m}(s)\right)$ converges to $f(s, u(s))$ in $X$, and we have

$$
\begin{gathered}
\left\|Q_{n 4} u_{m}-Q_{n 4} u\right\|_{\mathrm{PC}} \leq M \int_{0}^{K}\left\|f\left(s, u_{m}(s)\right)-f(s, u(s))\right\| \mathrm{d} s, \\
\left\|Q_{n 1} u_{m}-Q_{n 1} u\right\|_{\mathrm{PC}} \leq M\left\|g\left(u_{m}\right)-g(u)\right\|, \\
\left\|Q_{n 2} u_{m}-Q_{n 2} u\right\|_{\mathrm{PC}} \leq M \sum_{i=1}^{p}\left\|I_{i}\left(u_{m}\left(t_{i}\right)\right)-I_{i}\left(u\left(t_{i}\right)\right)\right\| .
\end{gathered}
$$

Then by the continuity of $f, g, I_{i}$, and using the dominated convergence theorem, we get

$$
\lim _{m \rightarrow \infty} Q_{n 4} u_{m}=Q_{n 4} u, \quad \lim _{m \rightarrow \infty} Q_{n 1} u_{m}=Q_{n 1} u, \quad \lim _{m \rightarrow \infty} Q_{n 2} u_{m}=Q_{n 2} u
$$

in $\operatorname{PC}([0, K], X)$, which implies that $Q_{n 4}, Q_{n 1}, Q_{n 2}$ are continuous on $\operatorname{PC}([0, K], X)$.

Next, for the compactness of $Q_{n 4}$ we refer to the proof of [4, Theorem 3.1].

For $Q_{n 1}$ and any bounded subset $W$ of $\mathrm{PC}([0, K], X)$, we have

$$
\left(Q_{n 1} u\right)(t)=T(t) u_{0}-T\left(\frac{1}{n}\right) T(t) g(u), \quad t \in[0, K], u \in W,
$$

which implies that $\left(Q_{n 1} W\right)(t)$ is relatively compact in $X$ for every $t \in[0, K]$ by the compactness of $T(1 / n)$. On the other hand, for $0 \leq s \leq t \leq K$, we have

$$
\left\|\left(Q_{n 1} u\right)(t)-\left(Q_{n 1} u\right)(s)\right\| \leq\left\|[T(t)-T(s)]\left[u_{0}-T\left(\frac{1}{n}\right) g(u)\right]\right\| .
$$

Since $\{T(1 / n) g(u) ; u \in W\}$ is relatively compact in $X$, we conclude that

$$
\left\|\left(Q_{n 1} u\right)(t)-\left(Q_{n 1} u\right)(s)\right\| \longrightarrow 0 \quad \text { uniformly as } t \longrightarrow s \text { and } u \in W \text {, }
$$

which implies that $Q_{n 1} W$ is equicontinuous on $[0, K]$. Therefore, $Q_{n 1}$ is a compact operator. Now, we prove the compactness of $Q_{n 2}$. For this purpose, let

$$
J_{0}=\left(0, t_{1}\right], J_{1}=\left(t_{1}, t_{2}\right], \ldots, J_{p}=\left(t_{p}, K\right] .
$$


Note that

$$
\left(Q_{n 2} u\right)(t)= \begin{cases}0, & t \in J_{0}, \\ T\left(t-t_{1}\right) T\left(\frac{1}{n}\right) I_{1}\left(u\left(t_{1}\right)\right), & t \in J_{1}, \\ \cdots & \\ \sum_{i=1}^{p} T\left(t-t_{i}\right) T\left(\frac{1}{n}\right) I_{i}\left(u\left(t_{i}\right)\right), & t \in J_{p} .\end{cases}
$$

Thus according to Lemma 2.3, we only need to prove that

$$
\left.\left\{Q_{n 2} u ; u \in W\right\}\right|_{\bar{J}_{1}}=\left\{T\left(\cdot-t_{1}\right) T\left(\frac{1}{n}\right) I_{1}\left(u\left(t_{1}\right)\right) ; \cdot \in \bar{J}_{1}, u \in W\right\}
$$

is precompact in $C\left(\left[t_{1}, t_{2}\right], X\right)$, as the remaining cases for $t \in \bar{J}_{i}, i=2,3, \ldots, p$, can be dealt with in the same way; here $W$ is any bounded subset in $\mathrm{PC}([0, K], X)$. And, we recall that $v=\left.\left(Q_{n_{2}} u\right)\right|_{\bar{J}_{1}}, u \in W$, which means that

$$
\begin{gathered}
v\left(t_{1}\right)=\left(Q_{n 2} u\right)\left(t_{1}^{+}\right)=T\left(\frac{1}{n}\right) I_{1}\left(u\left(t_{1}\right)\right), \\
v(t)=\left(Q_{n 2} u\right)(t)=T\left(t-t_{1}\right) T\left(\frac{1}{n}\right) I_{1}\left(u\left(t_{1}\right)\right), \quad t \in J_{1} .
\end{gathered}
$$

Thus, by the compactness of $T(1 / n)$, we know that $\left.\left\{\left(Q_{n_{2}} u\right) ; u \in W\right\}\right|_{\bar{J}_{1}}(t)$ is relatively compact in $X$ for every $t \in \bar{J}_{1}$.

Next, for $t_{1} \leq s \leq t \leq t_{2}$, we have

$$
\begin{gathered}
\left\|T\left(t-t_{1}\right) T\left(\frac{1}{n}\right) I_{1}\left(u\left(t_{1}\right)\right)-T\left(s-t_{1}\right) T\left(\frac{1}{n}\right) I_{1}\left(u\left(t_{1}\right)\right)\right\| \\
\quad=\left\|T\left(s-t_{1}\right)[T(t-s)-T(0)] T\left(\frac{1}{n}\right) I_{1}\left(u\left(t_{1}\right)\right)\right\| \\
\quad \leq M\left\|[T(t-s)-T(0)] T\left(\frac{1}{n}\right) I_{1}\left(u\left(t_{1}\right)\right)\right\| .
\end{gathered}
$$

Thus, the set $\left.\left\{Q_{n 2} u ; u \in W\right\}\right|_{\bar{J}_{1}} \subseteq C\left(\left[t_{1}, t_{2}\right], X\right)$ is equicontinuous due to the compactness of $\left\{T(1 / n) I_{1}\left(u\left(t_{1}\right)\right) ; u \in W\right\}$ and the strong continuity of operator $T(\cdot)$. By the Arzela-Ascoli theorem, we conclude that $\left.\left\{Q_{n 2} u ; u \in W\right\}\right|_{\bar{J}_{1}}$ is precompact in $C\left(\left[t_{1}, t_{2}\right], X\right)$. The same idea can be used to prove that $\left.\left\{Q_{n 2} u ; u \in W\right\}\right|_{J_{i}}$ is precompact for each $i=2,3, \ldots, p$. Therefore, $\left\{Q_{n 2} u ; u \in W\right\}$ is precompact in $\operatorname{PC}([0, K], X)$, that is, the operator $Q_{n 2}: \operatorname{PC}([0, K], X) \rightarrow$ $\mathrm{PC}([0, K], X)$ is compact. 
Thus, for any bounded subset $W \subseteq \mathrm{PC}([0, K], X)$, we have by Lemma 2.4,

$$
\alpha\left(Q_{n} W\right) \leq \alpha\left(Q_{n 1} W\right)+\alpha\left(Q_{n 3} W\right)+\alpha\left(Q_{n 4} W\right)+\alpha\left(Q_{n 2} W\right) \leq L_{0} \alpha(W)
$$

Hence, the map $Q_{n}$ is an $\alpha$-contraction in $\mathrm{PC}([0, K], X)$.

Now, in order to apply Lemma 2.5, it remains to prove that there exists a constant $r>0$ such that $Q_{n} W_{r} \subseteq W_{r}$. Suppose this is not true; then for each positive integer $r$, there are $u_{r} \in W_{r}$ and $t^{r} \in[0, K]$ such that $\left\|\left(Q_{n} u_{r}\right)\left(t^{r}\right)\right\|>r$. Then

$$
\begin{aligned}
r< & \left\|\left(Q_{n} u_{r}\right)\left(t^{r}\right)\right\| \\
= & \| T\left(t^{r}\right)\left[u_{0}-T\left(\frac{1}{n}\right) g\left(u_{r}\right)+F\left(0, u_{r}(0)\right)\right]-F\left(t^{r}, u_{r}\left(t^{r}\right)\right)+\int_{0}^{t^{r}} A T\left(t^{r}-s\right) F\left(s, u_{r}(s)\right) \mathrm{d} s \\
& \quad+\int_{0}^{t^{r}} T\left(t^{r}-s\right) f\left(s, u_{r}(s)\right) \mathrm{d} s+\sum_{0<t_{i}<t^{r}} T\left(t^{r}-t_{i}\right) T\left(\frac{1}{n}\right) I_{i}\left(u_{r}\left(t_{i}\right)\right) \| \\
\leq & M\left[\left\|u_{0}\right\|+L r+L_{1}^{\prime}+M_{0} L(r+1)\right]+M_{0} L(r+1)+\int_{0}^{t} \frac{C_{1-\beta}}{(t-s)^{1-\beta}} L(r+1) \mathrm{d} s \\
& +M \int_{0}^{t} \rho_{r}(s) \mathrm{d} s+M p\left(L r+L_{4}^{\prime}\right) \\
\leq & M\left(\left\|u_{0}\right\|+L r+L_{1}^{\prime}\right)+(1+M) M_{0} L(r+1)+\frac{L C_{1-\beta} K^{\beta}}{\beta}(r+1) \\
& +M \int_{0}^{K} \rho_{r}(s) \mathrm{d} s+M p\left(L r+L_{4}^{\prime}\right) .
\end{aligned}
$$

Dividing on both sides by $r$ and taking the lower limit as $r \rightarrow+\infty$, we obtain that

$$
L_{0}=M\left(L+M_{0} L+\gamma+p L\right)+M_{0} L+\frac{L C_{1-\beta} K^{\beta}}{\beta} \geq 1 .
$$

This is a contradiction with inequality (3.6). Therefore, there exists $r>0$ such that the mapping $Q_{n}$ maps $W_{r}$ into itself. By Darbu-Sadovskii's fixed point theorem, the operator $Q_{n}$ has at least one fixed point in $W_{r}$. This completes the proof.

Lemma 3.3. Assume that all the conditions in Theorem 3.1 are satisfied. Then the set $\left.D\right|_{[h, K]}$ is precompact in $\operatorname{PC}([h, K], X)$ for all $h \in(0, \delta)$, where

$$
D:=\left\{u_{n} ; u_{n} \in P C([0, K], X) \text { coming from Lemma 3.2, } n \geq 1\right\},
$$

and $\delta$ is the constant in (H2).

Proof. The proof will be given in several steps. In the following $h$ is a number in $(0, \delta)$. 
Step 1. $\left.D\right|_{\left[h, t_{1}\right]}$ is precompact in $C\left(\left[h, t_{1}\right], X\right)$.

For $u \in \mathrm{PC}([0, K], X)$, define $Q_{F 1}: \mathrm{PC}([0, K], X) \rightarrow \mathrm{PC}([0, K], X)$ by

$$
\left(Q_{F 1} u\right)(t)=T(t) F(0, u(0)), \quad t \in[0, K]
$$

For $u \in C\left(\left[h, t_{1}\right], X\right)$, let $\bar{u}(t)=u(t), t \in\left[h, t_{1}\right], \bar{u}(t)=u(h), t \in[0, h]$, and we define $Q_{F 2}: C\left(\left[h, t_{1}\right], X\right) \rightarrow C\left(\left[h, t_{1}\right], X\right)$ by

$$
\left(Q_{F 2} u\right)(t)=-F(t, u(t))+\int_{0}^{t} A T(t-s) F(s, \bar{u}(s)) \mathrm{d} s, \quad t \in\left[h, t_{1}\right]
$$

By condition (H3), $Q_{F 2}$ is well defined and for $u \in D$, we have

$$
\left(Q_{n 3} u\right)(t)=\left(Q_{F 1} u\right)(t)+\left(\left.Q_{F 2} u\right|_{\left[h, t_{1}\right]}\right)(t), \quad t \in\left[h, t_{1}\right]
$$

On the other hand, for $u_{n} \in D, n \geq 1$, we have $\left(Q_{n 2} u_{n}\right)(t)=0, t \in\left[h, t_{1}\right]$. So,

$$
u_{n}(t)=\left(Q_{n 1} u_{n}\right)(t)+\left(Q_{F 1} u_{n}\right)(t)+\left(\left.Q_{F 2} u_{n}\right|_{\left[h, t_{1}\right]}\right)(t)+\left(Q_{n 4} u_{n}\right)(t), \quad t \in\left[h, t_{1}\right]
$$

Now, for $\left\{Q_{n 1} u_{n} ; n \geq 1\right\}$, we have

$$
\left(Q_{n 1} u_{n}\right)(t)=T(t) u_{0}-T(t) T\left(\frac{1}{n}\right) g\left(u_{n}\right), \quad t \in\left[h, t_{1}\right]
$$

By the compactness of $T(t), t>0$, we get that $\left\{\left(Q_{n 1} u_{n}\right)(t) ; n \geq 1\right\}$ is relatively compact in $X$ for every $t \in\left[h, t_{1}\right]$ and $\left.\left\{Q_{n 1} u_{n} ; n \geq 1\right\}\right|_{\left[h, t_{1}\right]}$ is equicontinuous on $\left[h, t_{1}\right]$, which implies that $\left.\left\{Q_{n 1} u_{n} ; n \geq 1\right\}\right|_{\left[h, t_{1}\right]}$ is precompact in $C\left(\left[h, t_{1}\right], X\right)$.

By the same reasoning, $\left.\left\{Q_{F 1} u_{n} ; n \geq 1\right\}\right|_{\left[h, t_{1}\right]}$ is precompact in $C\left(\left[h, t_{1}\right], X\right)$.

For $Q_{F 2}$, we claim that $Q_{F 2}: C\left(\left[h, t_{1}\right], X\right) \rightarrow C\left(\left[h, t_{1}\right], X\right)$ is Lipschitz continuous with constant $M_{0} L+\left(L C_{1-\beta} K^{\beta}\right) / \beta$. In fact, (H3) implies that for every $u, v \in C\left(\left[h, t_{1}\right], X\right)$ and $t \in\left[h, t_{1}\right]$,

$$
\begin{aligned}
& \left\|\left(Q_{F 2} u\right)(t)-\left(Q_{F 2} v\right)(t)\right\| \\
& \quad \leq\|F(t, u(t))-F(t, v(t))\|+\int_{0}^{t}\|A T(t-s)[F(s, \bar{u}(s))-F(s, \bar{v}(s))]\| \mathrm{d} s \\
& \quad \leq M_{0} L\|u(t)-v(t)\|+\int_{0}^{t} \frac{C_{1-\beta}}{(t-s)^{1-\beta}} L \mathrm{~d} s \max _{0 \leq t \leq t_{1}}\|\bar{u}(t)-\bar{v}(t)\| \\
& \quad \leq M_{0} L\|u(t)-v(t)\|+\frac{L C_{1-\beta} K^{\beta}}{\beta} \max _{h \leq t \leq t_{1}}\|u(t)-v(t)\|,
\end{aligned}
$$


that is,

$$
\left\|Q_{F 2} u-Q_{F 2} v\right\|_{C\left(\left[h, t_{1}\right], X\right)} \leq\left(M_{0} L+\frac{L C_{1-\beta} K^{\beta}}{\beta}\right)\|u-v\|_{C\left(\left[h, t_{1}\right], X\right)}
$$

Therefore, $Q_{F 2}: C\left(\left[h, t_{1}\right], X\right) \rightarrow C\left(\left[h, t_{1}\right], X\right)$ is Lipschitz continuous with constant $M_{0} L+$ $\left(L C_{1-\beta} K^{\beta}\right) / \beta$.

Clearly, $\left\{Q_{n 4} u_{n} ; n \geq 1\right\}$ is precompact in $\mathrm{PC}([0, K], X)$, and so is $\left.\left\{Q_{n 4} u_{n} ; n \geq 1\right\}\right|_{\left[h, t_{1}\right]}$ in $C\left(\left[h, t_{1}\right], X\right)$.

Thus, by (3.29) and Lemma 2.4, we obtain

$$
\alpha\left(\left.D\right|_{\left[h, t_{1}\right]}\right) \leq\left(M_{0} L+\frac{L C_{1-\beta} K^{\beta}}{\beta}\right) \alpha\left(\left.D\right|_{\left[h, t_{1}\right]}\right) .
$$

By (3.6), $\left(M_{0} L+\left(L C_{1-\beta} K^{\beta}\right) / \beta\right)<1$, which implies $\alpha\left(\left.D\right|_{\left[h, t_{1}\right]}\right)=0$. Consequently, $\left.D\right|_{\left[h, t_{1}\right]}$ is precompact in $C\left(\left[h, t_{1}\right], X\right)$.

Step 2. $\left.D\right|_{\left[h, t_{2}\right]}$ is precompact in $\operatorname{PC}\left(\left[h, t_{2}\right], X\right)$.

For $u \in \mathrm{PC}\left(\left[h, t_{2}\right], X\right)$, let

$$
\bar{u}(t)=u(t), \quad t \in\left[h, t_{2}\right], \quad \bar{u}(t)=u(h), \quad t \in[0, h],
$$

and define $Q_{F 2}^{\prime}: \operatorname{PC}\left(\left[h, t_{2}\right], X\right) \rightarrow \mathrm{PC}\left(\left[h, t_{2}\right], X\right)$ by

$$
\left(Q_{F 2}^{\prime} u\right)(t)=-F(t, u(t))+\int_{0}^{t} A T(t-s) F(s, \bar{u}(s)) \mathrm{d} s, \quad t \in\left[h, t_{2}\right]
$$

By (H3), $Q_{F 2}^{\prime}$ is well defined and for $u \in D$, we have

$$
\left(Q_{n 3} u\right)(t)=\left(Q_{F 1} u\right)(t)+\left(\left.Q_{F 2}^{\prime} u\right|_{\left[h, t_{2}\right]}\right)(t), \quad t \in\left[h, t_{2}\right] .
$$

So, for $u_{n} \in D, n \geq 1$, we have

$$
u_{n}(t)=\left(Q_{n 1} u_{n}\right)(t)+\left(Q_{F 1} u_{n}\right)(t)+\left(\left.Q_{F 2}^{\prime} u_{n}\right|_{\left[h, t_{2}\right]}\right)(t)+\left(Q_{n 4} u_{n}\right)(t)+\left(Q_{n 2} u_{n}\right)(t), \quad t \in\left[h, t_{2}\right],
$$

where

$$
\left(Q_{n 2} u_{n}\right)(t)= \begin{cases}0, & t \in\left[h, t_{1}\right], \\ T\left(t-t_{1}\right) T\left(\frac{1}{n}\right) I_{1}\left(u_{n}\left(t_{1}\right)\right), & t \in J_{1} .\end{cases}
$$


According to the proof of Step 1, we know that

$$
\left.\left\{Q_{n 1} u_{n} ; n \geq 1\right\}\right|_{\left[h, t_{2}\right]},\left.\quad\left\{Q_{F 1} u_{n} ; n \geq 1\right\}\right|_{\left[h, t_{2}\right]},\left.\quad\left\{Q_{n 4} u_{n} ; n \geq 1\right\}\right|_{\left[h, t_{2}\right]}
$$

are all precompact in $\mathrm{PC}\left(\left[h, t_{2}\right], X\right)$ and $Q_{F 2}^{\prime}: \mathrm{PC}\left(\left[h, t_{2}\right], X\right) \rightarrow \mathrm{PC}\left(\left[h, t_{2}\right], X\right)$ is Lipschitz continuous with constant $M_{0} L+\left(L C_{1-\beta} K^{\beta}\right) / \beta$.

Next, we will show that $\left.\left\{Q_{n 2} u_{n} ; n \geq 1\right\}\right|_{\left[h, t_{2}\right]}$ is precompact in $\operatorname{PC}\left(\left[h, t_{2}\right], X\right)$. Firstly, it is easy to see that $\left.\left\{Q_{n 2} u_{n} ; n \geq 1\right\}\right|_{\left[h, t_{1}\right]}$ is precompact in $C\left(\left[h, t_{1}\right], X\right)$. Thus according to Lemma 2.3, it remains to prove that

$$
\left.\left\{Q_{n 2} u_{n} ; n \geq 1\right\}\right|_{\bar{J}_{1}}=\left\{T\left(\cdot-t_{1}\right) T\left(\frac{1}{n}\right) I_{1}\left(u_{n}\left(t_{1}\right)\right) ; \cdot \in \bar{J}_{1}, n \geq 1\right\}
$$

is precompact in $C\left(\left[t_{1}, t_{2}\right], X\right)$. And, we recall that $v_{n}=\left.\left(Q_{n 2} u_{n}\right)\right|_{\bar{J}_{1}}, n \geq 1$, which means that

$$
\begin{gathered}
v_{n}\left(t_{1}\right)=\left(Q_{n 2} u_{n}\right)\left(t_{1}^{+}\right)=T\left(\frac{1}{n}\right) I_{1}\left(u_{n}\left(t_{1}\right)\right), \\
v_{n}(t)=\left(Q_{n 2} u_{n}\right)(t)=T\left(t-t_{1}\right) T\left(\frac{1}{n}\right) I_{1}\left(u_{n}\left(t_{1}\right)\right), \quad t \in J_{1} .
\end{gathered}
$$

By Step $1,\left.D\right|_{\left[h, t_{1}\right]}$ is precompact in $C\left(\left[h, t_{1}\right], X\right)$. Without loss of generality, we may suppose that

$$
\left.u_{n}\right|_{\left[h, t_{1}\right]} \longrightarrow w, \quad \text { as } n \longrightarrow \infty \text { in } C\left(\left[h, t_{1}\right], X\right)
$$

Therefore, $u_{n}\left(t_{1}\right) \rightarrow w\left(t_{1}\right)$, as $n \rightarrow \infty$ in $X$. Thus, by the continuity of $I_{1}$ and $T(t)$, we get

$$
\begin{aligned}
& \left\|T\left(\frac{1}{n}\right) I_{1}\left(u_{n}\left(t_{1}\right)\right)-I_{1}\left(w\left(t_{1}\right)\right)\right\| \\
& \quad \leq\left\|T\left(\frac{1}{n}\right) I_{1}\left(u_{n}\left(t_{1}\right)\right)-T\left(\frac{1}{n}\right) I_{1}\left(w\left(t_{1}\right)\right)\right\|+\left\|T\left(\frac{1}{n}\right) I_{1}\left(w\left(t_{1}\right)\right)-I_{1}\left(w\left(t_{1}\right)\right)\right\| \\
& \quad \leq M\left\|I_{1}\left(u_{n}\left(t_{1}\right)\right)-I_{1}\left(w\left(t_{1}\right)\right)\right\|+\left\|T\left(\frac{1}{n}\right) I_{1}\left(w\left(t_{1}\right)\right)-I_{1}\left(w\left(t_{1}\right)\right)\right\| \\
& \quad \longrightarrow 0
\end{aligned}
$$

as $n \rightarrow \infty$, which implies that $\left\{v_{n}\left(t_{1}\right) ; n \geq 1\right\}$ is relatively compact in $X$. And, for $t \in J_{1}$, by the compactness of $T(t), t>0,\left\{v_{n}(t) ; n \geq 1\right\}$ is also relatively compact in $X$. Therefore, $\left.\left\{Q_{n 2} u_{n} ; n \geq 1\right\}\right|_{\bar{J}_{1}}(t)$ is relatively compact in $X$ for every $t \in \overline{J_{1}}$. 
Next, for $t_{1} \leq s \leq t \leq t_{2}$, we have

$$
\begin{gathered}
\left\|T\left(t-t_{1}\right) T\left(\frac{1}{n}\right) I_{1}\left(u_{n}\left(t_{1}\right)\right)-T\left(s-t_{1}\right) T\left(\frac{1}{n}\right) I_{1}\left(u_{n}\left(t_{1}\right)\right)\right\| \\
\quad=\left\|T\left(s-t_{1}\right)[T(t-s)-T(0)] T\left(\frac{1}{n}\right) I_{1}\left(u_{n}\left(t_{1}\right)\right)\right\| \\
\quad \leq M\left\|[T(t-s)-T(0)] T\left(\frac{1}{n}\right) I_{1}\left(u_{n}\left(t_{1}\right)\right)\right\| .
\end{gathered}
$$

Thus, the set $\left.\left\{Q_{n 2} u_{n} ; n \geq 1\right\}\right|_{\bar{J}_{1}} \subseteq C\left(\left[t_{1}, t_{2}\right], X\right)$ is equicontinuous on $\bar{J}_{1}$ due to the compactness of $\left\{T(1 / n) I_{1}\left(u_{n}\left(t_{1}\right)\right) ; n \geq 1\right\}$ and the strong continuity of operator $T(t), t \geq 0$. By the ArzelaAscoli theorem, we conclude that $\left.\left\{Q_{n 2} u_{n} ; n \geq 1\right\}\right|_{\bar{J}_{1}}$ is precompact in $C\left(\left[t_{1}, t_{2}\right], X\right)$. Therefore, $\left.\left\{Q_{n 2} u_{n} ; n \geq 1\right\}\right|_{\left[h, t_{2}\right]}$ is precompact in $\operatorname{PC}\left(\left[h, t_{2}\right], X\right)$.

Thus, by Lemma 2.4, we obtain

$$
\alpha\left(\left.D\right|_{\left[h, t_{2}\right]}\right) \leq\left(M_{0} L+\frac{L C_{1-\beta} K^{\beta}}{\beta}\right) \alpha\left(\left.D\right|_{\left[h, t_{2}\right]}\right) .
$$

By (3.6), $\left(M_{0} L+\left(L C_{1-\beta} K^{\beta}\right) / \beta\right)<1$, which implies $\alpha\left(\left.D\right|_{\left[h, t_{2}\right]}\right)=0$. Consequently, $\left.D\right|_{\left[h, t_{2}\right]}$ is precompact in $\mathrm{PC}\left(\left[h, t_{2}\right], X\right)$.

Step 3. The same idea can be used to prove the compactness of $\left.D\right|_{\left[h, t_{i}\right]}$ in $\operatorname{PC}\left(\left[h, t_{i}\right], X\right)$ for $i=3, \ldots, p, p+1$, where $t_{p+1}=K$. This completes the proof.

Proof of Theorem 3.1. For $u_{n} \in D, n \geq 1$, let

$$
\bar{u}_{n}(t)= \begin{cases}u_{n}(t), & t \in[\delta, K], \\ u_{n}(\delta), & t \in[0, \delta],\end{cases}
$$

where $\delta$ comes from the condition (H2). Then, by condition (H2), $g\left(u_{n}\right)=g\left(\bar{u}_{n}\right)$.

By Lemma 3.3, without loss of generality, we may suppose that $\bar{u}_{n} \rightarrow u \in$ $\mathrm{PC}([0, K], X)$, as $n \rightarrow \infty$. Thus, by the continuity of $T(t)$ and $g$, we get

$$
\begin{aligned}
& \left\|T\left(\frac{1}{n}\right) g\left(u_{n}\right)-g(u)\right\| \\
& \quad \leq\left\|T\left(\frac{1}{n}\right) g\left(u_{n}\right)-T\left(\frac{1}{n}\right) g(u)\right\|+\left\|T\left(\frac{1}{n}\right) g(u)-g(u)\right\| \\
& \quad \leq M\left\|g\left(\bar{u}_{n}\right)-g(u)\right\|+\left\|T\left(\frac{1}{n}\right) g(u)-g(u)\right\| \\
& \quad \longrightarrow 0
\end{aligned}
$$


as $n \rightarrow \infty$. Thus,

$$
\left\{Q_{n 1} u_{n} ; n \geq 1\right\}=\left\{T(\cdot)\left[u_{0}-T\left(\frac{1}{n}\right) g\left(u_{n}\right)\right] ; n \geq 1\right\}
$$

is precompact in $\mathrm{PC}([0, K], X)$. Moreover, $\left\{Q_{n 4} u_{n} ; n \geq 1\right\}$ and $\left\{Q_{n 2} u_{n} ; n \geq 1\right\}$ are both precompact in $\mathrm{PC}([0, K], X)$. And $Q_{n 3}: \mathrm{PC}([0, K], X) \rightarrow \mathrm{PC}([0, K], X)$ is Lipschitz continuous with constant $(M+1) M_{0} L+\left(L C_{1-\beta} K^{\beta}\right) / \beta$. Note that

$$
u_{n}(t)=\left(Q_{n} u_{n}\right)(t)=\left(Q_{n 1} u_{n}\right)(t)+\left(Q_{n 3} u_{n}\right)(t)+\left(Q_{n 4} u_{n}\right)(t)+\left(Q_{n 2} u_{n}\right)(t), \quad t \in[0, K]
$$

Therefore, by Lemma 2.4, we know that the set $D$ is precompact in $\operatorname{PC}([0, K], X)$. Without loss of generality, we may suppose that $u_{n} \rightarrow u^{*}$ in $\mathrm{PC}([0, K], X)$. On the other hand, we also have

$$
\begin{aligned}
u_{n}(t)= & T(t)\left[u_{0}+F\left(0, u_{n}(0)\right)-T\left(\frac{1}{n}\right) g\left(u_{n}\right)\right]-F\left(t, u_{n}(t)\right) \\
& +\int_{0}^{t} A T(t-s) F\left(s, u_{n}(s)\right) \mathrm{d} s+\int_{0}^{t} T(t-s) f\left(s, u_{n}(s)\right) \mathrm{d} s \\
& +\sum_{0<t_{i}<t} T\left(t-t_{i}\right) T\left(\frac{1}{n}\right) I_{i}\left(u_{n}\left(t_{i}\right)\right), \quad 0 \leq t \leq K .
\end{aligned}
$$

Letting $n \rightarrow \infty$ in both sides, we obtain

$$
\begin{aligned}
u^{*}(t)= & T(t)\left[u_{0}+F\left(0, u^{*}(0)\right)-g\left(u^{*}\right)\right]-F\left(t, u^{*}(t)\right) \\
& +\int_{0}^{t} A T(t-s) F\left(s, u^{*}(s)\right) \mathrm{d} s+\int_{0}^{t} T(t-s) f\left(s, u^{*}(s)\right) \mathrm{d} s \\
& +\sum_{0<t_{i}<t} T\left(t-t_{i}\right) I_{i}\left(u^{*}\left(t_{i}\right)\right), \quad 0 \leq t \leq K,
\end{aligned}
$$

which implies that $u^{*}$ is a mild solution of the nonlocal impulsive problem (1.1). This completes the proof.

Remark 3.4. From Lemma 3.3 and the above proof, it is easy to see that we can also prove Theorem 3.1 by showing that $\left.D\right|_{[0, h]}$ is precompact in $\mathrm{PC}([0, h], X)$.

The following results are immediate consequences of Theorem 3.5.

Theorem 3.5. Assume (H1), (H3)-(H5) hold. If $g \equiv 0$, then the impulsive Cauchy problem (1.1) has at least one mild solution on $[0, K]$, provided

$$
M\left(M_{0} L+\gamma+p L\right)+M_{0} L+\frac{L C_{1-\beta} K^{\beta}}{\beta}<1
$$


Theorem 3.6. Assume (H1), (H2), (H4), and (H5) hold. If $F \equiv 0$, then the nonlocal impulsive problem (1.1) has at least one mild solution on $[0, K]$, provided $M(L+\gamma+p L)<1$.

Theorem 3.7. Assume (H1), (H4), and (H5) hold. If $g \equiv 0, F \equiv 0$, then the impulsive problem (1.1) has at least one mild solution on $[0, K]$, provided $M(\gamma+p L)<1$.

Remark 3.8. Theorems 3.5-3.6 are new even for many special cases discussed before, since neither the Lipschitz continuity nor compactness assumption on the impulsive functions is required.

\section{Application}

In this section, to illustrate our abstract result, we consider the following differential system:

$$
\begin{array}{r}
\frac{\partial}{\partial t}\left[w(t, x)+\int_{0}^{\pi} \lambda(t, x, y) w(t, y) \mathrm{d} y\right]=\frac{\partial^{2}}{\partial x^{2}} w(t, x)+v(t, w(t, x)), \\
0 \leq t \leq 1,0 \leq x \leq \pi, t \neq t_{i}, \\
w(t, 0)=w(t, \pi)=0, \quad 0 \leq t \leq 1, \\
w\left(t_{i}^{+}\right)-w\left(t_{i}^{-}\right)=I_{i}\left(w\left(t_{i}\right)\right), \quad i=1, \ldots, p, 0<t_{1}<\cdots<t_{p}<1, \\
w(0, x)+\sum_{j=1}^{q} c_{j} w\left(s_{j}, x\right)=w_{0}(x), \quad 0<s_{1}<\cdots<s_{q}<1,0 \leq x \leq \pi,
\end{array}
$$

where $w_{0} \in L^{2}([0, \pi]), t_{i}, s_{j}, c_{j}$ are given real numbers for $i=1, \ldots, p, j=1, \ldots, q$, and $\lambda$ : $[0,1] \times[0, \pi] \times[0, \pi] \rightarrow \mathbb{R}$ and $v:[0,1] \times \mathbb{R} \rightarrow \mathbb{R}$ are functions to be specified below.

To treat the above system, we take $X=L^{2}([0, \pi])$ with the norm $\|\cdot\|$ and we consider the operator $A: D(A) \subseteq X \rightarrow X$ defined by

$$
A z=-z^{\prime \prime}
$$

with domain

$$
D(A)=\left\{z \in X ; z, z^{\prime} \text { area absolutely continuous, } z^{\prime \prime} \in X, z(0)=z(\pi)=0\right\} .
$$

The operator $-A$ is the infinitesimal generator of an analytic compact semigroup $(T(t))_{t>0}$ on $X$. Moreover, $A$ has a discrete spectrum, the eigenvalues are $n^{2}, n \in \mathbb{N}$, with the corresponding normalized eigenvectors $e_{n}(x)=\sqrt{2 / \pi} \sin (n x)$, and the following properties are satisfied.

(a) If $z \in D(A)$, then $A z=\sum_{n=1}^{\infty} n^{2}\left\langle z, e_{n}\right\rangle e_{n}$.

(b) For each $z \in X, T(t) z=\sum_{n=1}^{\infty} \exp \left(-n^{2} t\right)\left\langle z, e_{n}\right\rangle e_{n}$. Moreover, $\|T(t)\| \leq 1$ for all $t \geq 0$.

(c) For each $z \in X, A^{-1 / 2} z=\sum_{n=1}^{\infty} 1 / n\left\langle z, e_{n}\right\rangle e_{n}$. In particular, $\left\|A^{-1 / 2}\right\|=1$.

(d) $A^{1 / 2}$ is given by $A^{1 / 2} z=\sum_{n=1}^{\infty} n\left\langle z, e_{n}\right\rangle e_{n}$ with the domain $D\left(A^{1 / 2}\right)=\{z \in$ $\left.X ; \sum_{n=1}^{\infty} n\left\langle z, e_{n}\right\rangle e_{n} \in X\right\}$. 
Assume the following.

(1) The function $\lambda:[0,1] \times[0, \pi] \times[0, \pi] \rightarrow \mathbb{R}$ is continuously differential with $\lambda(t, 0, y)=\lambda(t, \pi, y)=0$ for $t \in[0,1], y \in[0, \pi]$, and there exists a real number $\delta \in\left(0, s_{1}\right)$ such that $\lambda(t, x, y)=0$ for $t \in[0, \delta], x, y \in[0, \pi]$. Moreover,

$$
\Lambda:=\sup _{t \in[0,1]}\left[\iint_{0}^{\pi}\left(\left(\frac{\partial}{\partial x}\right) \lambda(t, x, y)\right)^{2} \mathrm{~d} x \mathrm{~d} y\right]^{1 / 2}<\infty
$$

(2) For each $t \in[0,1], v(t, \cdot)$ is continuous, and for each $x \in \mathbb{R}, v(\cdot, x)$ is measurable and, there exists a function $a(\cdot) \in L^{1}([0,1], \mathbb{R})$ such that $|v(t, x)| \leq a(t)|x|$ for a.e. $t \in[0,1]$ and all $x \in \mathbb{R}$.

(3) $I_{i}: X \rightarrow X$ is a continuous function for each $i=1, \ldots, p$, and there exist positive numbers $L_{4}, L_{4}^{\prime}$ such that $\left\|I_{i}(z)\right\| \leq L_{4}\|z\|+L_{4}^{\prime}$ for any $z \in X$ and $i=1,2, \ldots, p$.

Define $F, f:[0,1] \times X \rightarrow X$ and $g: \mathrm{PC}([0,1], X) \rightarrow X$, respectively, as follows. For $x \in[0, \pi]$,

$$
\begin{gathered}
F(t, z)(x)=\int_{0}^{\pi} \lambda(t, x, y) z(y) \mathrm{d} y, \\
f(t, z)(x)=v(t, z(x)), \\
g(u)(x)=\sum_{j=1}^{q} c_{j} u\left(s_{j}\right)(x) .
\end{gathered}
$$

From the definition of $F$ and assumption (1), it follows that

$$
\begin{aligned}
F\left(\cdot, u_{1}(\cdot)\right)=F\left(\cdot, u_{2}(\cdot)\right) \quad \text { with } u_{1}(t)=u_{2}(t), t \in[\delta, 1], \text { for } u_{1}, u_{2} \in \operatorname{PC}([0,1], X), & \\
\left\langle F(t, z), e_{n}\right\rangle= & \int_{0}^{\pi} e_{n}(x)\left(\int_{0}^{\pi} \lambda(t, x, y) z(y) \mathrm{d} y\right) \mathrm{d} x \\
= & \frac{1}{n}\left\langle\int_{0}^{\pi} \frac{\partial}{\partial x} \lambda(t, x, y) z(y) \mathrm{d} y, \sqrt{\frac{2}{\pi}} \cos (n x)\right\rangle, \\
\left\|A^{1 / 2} F\left(t, z_{1}\right)-A^{1 / 2} F\left(t, z_{2}\right)\right\|= & \left.\left\|\sum_{n=1}^{\infty} n\left\langle F\left(t, z_{1}\right)-F\left(t, z_{2}\right), e_{n}\right\rangle e_{n}\right\|\right]^{1 / 2} \\
\leq & {\left[\iint_{0}^{\pi}\left(\left(\frac{\partial}{\partial x}\right) \lambda(t, x, y)\right)^{2} \mathrm{~d} y \mathrm{~d} x\right]^{1 / 2} } \\
& \times\left[\int_{0}^{\pi}\left(z_{1}(y)-z_{2}(y)\right)^{2} \mathrm{~d} y\right]^{1 / 2} \\
\leq & \Lambda\left\|z_{1}-z_{2}\right\| .
\end{aligned}
$$

Thus, system (4.1) can be transformed into the abstract problem (1.1), and conditions (H2), (H3), (H4), and (H5) are satisfied with

$$
L_{1}=\sum_{j=1}^{q}\left|c_{j}\right|, \quad L_{2}=L_{3}=\Lambda, \quad \rho_{l}(t)=l a(t), \quad \gamma=\int_{0}^{1} a(t) \mathrm{d} t
$$


If (3.6) holds (it holds when the related constants are small), then according to Theorem 3.1, the problem (4.1) has at least one mild solution in $\mathrm{PC}([0,1], X)$.

\section{Acknowledgments}

The authors would like to thank the referees for helpful comments and suggestions. J. Liang acknowledges support from the NSF of China (10771202) and the Specialized Research Fund for the Doctoral Program of Higher Education of China (2007035805). Z. Fan acknowledges support from the NSF of China (11001034) and the Research Fund for Shanghai Postdoctoral Scientific Program (10R21413700).

\section{References}

[1] N. U. Ahmed, "Optimal feedback control for impulsive systems on the space of finitely additive measures," Publicationes Mathematicae Debrecen, vol. 70, no. 3-4, pp. 371-393, 2007.

[2] T. Cardinali and P. Rubbioni, "Impulsive semilinear differential inclusions: topological structure of the solution set and solutions on non-compact domains," Nonlinear Analysis: Theory, Methods $\mathcal{E}$ Applications, vol. 69, no. 1, pp. 73-84, 2008.

[3] M. Eduardo Hernández and S. M. Tanaka Aki, "Global solutions for abstract impulsive differential equations," Nonlinear Analysis: Theory, Methods E Applications, vol. 72, no. 3-4, pp. 1280-1290, 2010.

[4] J. Liang, J. H. Liu, and T.-J. Xiao, "Nonlocal impulsive problems for nonlinear differential equations in Banach spaces," Mathematical and Computer Modelling, vol. 49, no. 3-4, pp. 798-804, 2009.

[5] J. H. Liu, "Nonlinear impulsive evolution equations," Dynamics of Continuous, Discrete and Impulsive Systems, vol. 6, no. 1, pp. 77-85, 1999.

[6] Y. V. Rogovchenko, "Impulsive evolution systems: main results and new trends," Dynamics of Continuous, Discrete and Impulsive Systems, vol. 3, no. 1, pp. 57-88, 1997.

[7] S. Aizicovici and H. Lee, "Nonlinear nonlocal Cauchy problems in Banach spaces," Applied Mathematics Letters, vol. 18, no. 4, pp. 401-407, 2005.

[8] S. Aizicovici and V. Staicu, "Multivalued evolution equations with nonlocal initial conditions in Banach spaces," NoDEA. Nonlinear Differential Equations and Applications, vol. 14, no. 3-4, pp. 361-376, 2007.

[9] L. Byszewski and V. Lakshmikantham, "Theorem about the existence and uniqueness of a solution of a nonlocal abstract Cauchy problem in a Banach space," Applicable Analysis, vol. 40, no. 1, pp. 11-19, 1991.

[10] J. Liang, J. Liu, and T.-J. Xiao, "Nonlocal Cauchy problems governed by compact operator families," Nonlinear Analysis: Theory, Methods E Applications, vol. 57, no. 2, pp. 183-189, 2004.

[11] G. M. Mophou and G. M. N'Guérékata, “Existence of the mild solution for some fractional differential equations with nonlocal conditions," Semigroup Forum, vol. 79, no. 2, pp. 315-322, 2009.

[12] T.-J. Xiao and J. Liang, "Existence of classical solutions to nonautonomous nonlocal parabolic problems," Nonlinear Analysis, Theory, Methods and Applications, vol. 63, no. 5-7, pp. e225-e232, 2005.

[13] A. Pazy, Semigroups of Linear Operators and Applications to Partial Differential Equations, vol. 44 of Applied Mathematical Sciences, Springer, New York, NY, USA, 1983.

[14] J. Banaś and K. Goebel, Measures of Noncompactness in Banach Spaces, vol. 60 of Lecture Notes in Pure and Applied Mathematics, Marcel Dekker, New York, NY, USA, 1980. 